\title{
¿̇Es posible decolonizar la extensión?: reflexiones acerca de la curricularización de la extensión y la integralidad en dos modelos universitarios
}

\author{
Romina Hortegano' \\ RECIBIDO: 30/01/2021; ACEPTADO: 18/05/2021 \\ DOI: https://doi.org/10.37125/ISR.7.1.2
}

\section{Resumen}

Con el propósito de reflexionar acerca de la curricularización de la extensión desde una perspectiva decolonial, este artículo desarrolla una comparación entre dos modelos específicos: el de la Universidad de la República en el marco de la segunda reforma, y el de la Universidad Bolivariana de Venezuela como parte del proceso de transformación universitaria. A los efectos, se recorre la historia de la universidad en América Latina, seguido del abordaje comparativo de los modelos seleccionados, para finalizar con un abordaje decolonial de ambas experiencias, que deja una serie de consideraciones al respecto.

Palabras clave: decolonización, curricularización, extensión

¿Qué sucederá si en la Universidad decenas de miles de estudiantes realizan actividades de extensión conectadas de manera natural con sus trayectorias educativas?

Rodrigo Arocena, «Curricularización de la extensión: ¿por qué, cuál, cómo?»

Cuando Humberto Tommasino y Agustín Cano en el 2016 afirman que «en nuestras universidades públicas [...] sobre la base del modelo profesionalista hegemónico a lo largo del siglo xx se ha consolidado progresivamente una concepción universitaria tecnocrática tendencialmente orientada al mercado» (p. 16), dan cuenta de un proceso de larga data que continúa vigente, aun cuando en América Latina existe una también larga tradición de luchas por transformar dicha concepción. 1 Unidad de Apoyo a la Enseñanza de la Facultad de Humanidades y Ciencias de la Educación (FHCE),
Universidad de la República (Udelar).romina.hortegano@fhce.edu.uy. 
Tales luchas, cuyo primer hito histórico es la Reforma de Córdoba de 1918, se inspiran en la meta de superar el modelo colonial de educación superior transferido a nuestro continente por parte de los poderes metropolitanos que nos habían dominado (Jiménez, 2007), y tienen un recorrido bastante complejo que ha tenido su incidencia directa en la forma de concebir la extensión, siendo este uno de los aportes más significativos de la universidad latinoamericana.

Trataremos en estas breves páginas, reflexionar un poco acerca de la curricularización de la extensión desde una perspectiva decolonial, no sin antes pasar de forma sucinta por una mirada histórica de la universidad en América Latina y las nociones y modelos de extensión vinculantes según tal contexto histórico; para luego detenernos en dos modelos concretos de extensión: el uruguayo de la segunda reforma universitaria y el venezolano en el contexto del proceso de transformación universitaria que cursa en la actualidad.

En tal sentido, la primera parte se centrará en un recorrido por los modelos de universidad y de extensión en América Latina; luego, se presentarán las aproximaciones, diferenciaciones y retos de los modelos de curricularización de la extensión en la Universidad de la República (Udelar) en Uruguay y la Universidad Bolivariana de Venezuela (UBV); para finalizar, se ofrecerán algunas consideraciones a modo de reflexiones que, en clave decolonial, ${ }^{2}$ se desprenden de este análisis.

\section{Contexto histórico de los modelos de universidad y extensión en América Latina}

Organizamos este recorrido en tres grandes bloques: universidad colonial, universidad republicana (siglos XVIII y XIX), universidad contemporánea. Tal organización solo tiene fines metodológicos a los efectos de orientar las reflexiones finales que nos motivan, no tienen carácter resolutorio de un asunto tan completo y variopinto como lo es la historia de nuestro continente y las dinámicas que atravesaron las universidades en este devenir. Para tales efectos, resumiremos los rasgos que más nos interesa destacar en el Cuadro 1.

2 Véase Mignolo (2008) acerca de lo que consideramos perspectiva decolonial. 
Cuadro 1.

Resumen histórico de la universidad en el devenir de América Latina

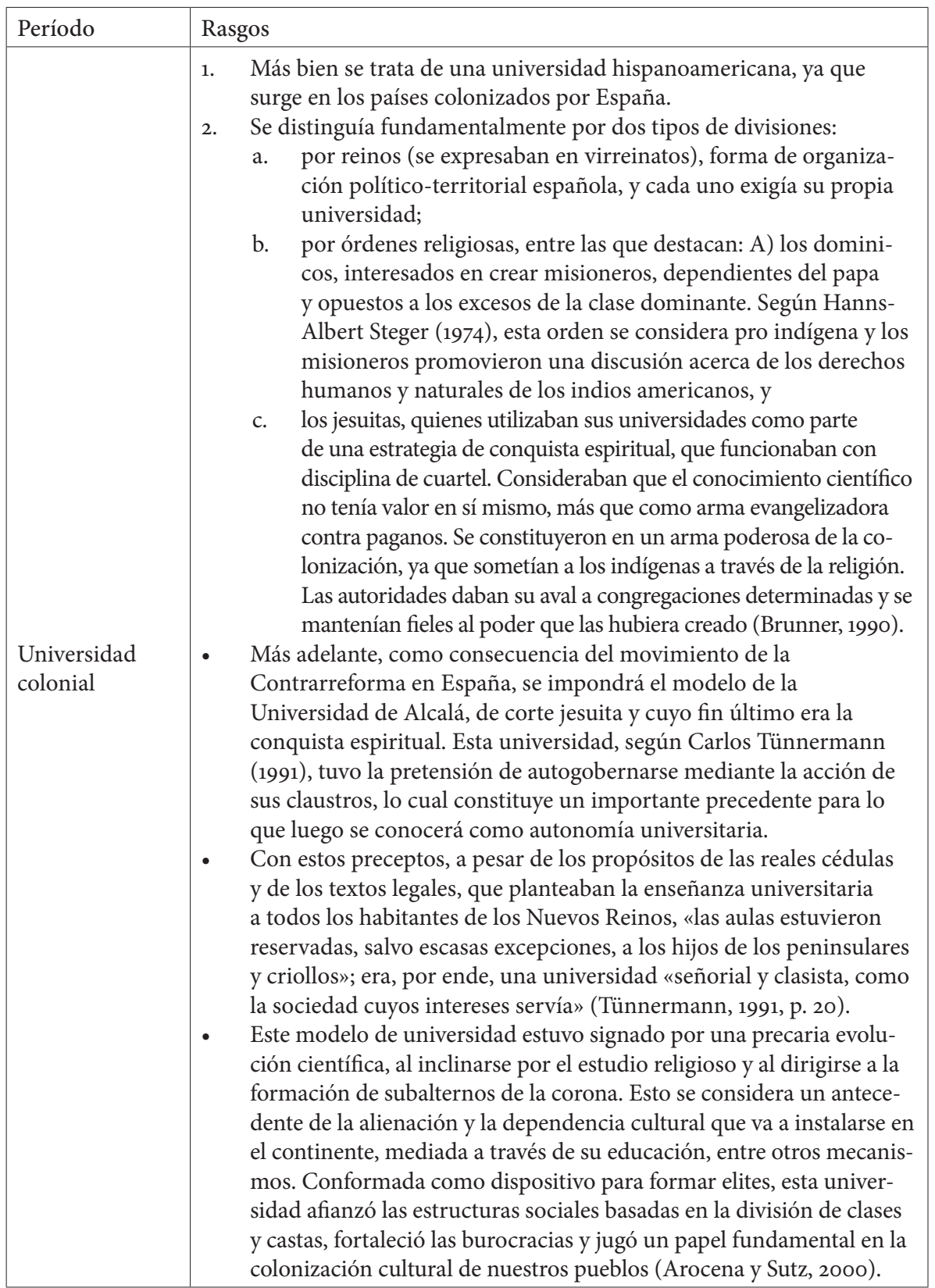




\begin{tabular}{|c|c|}
\hline Período & Rasgos \\
\hline $\begin{array}{l}\text { Universidad } \\
\text { republicana } \\
\text { (siglos XVIII y } \\
\text { XIX) }\end{array}$ & 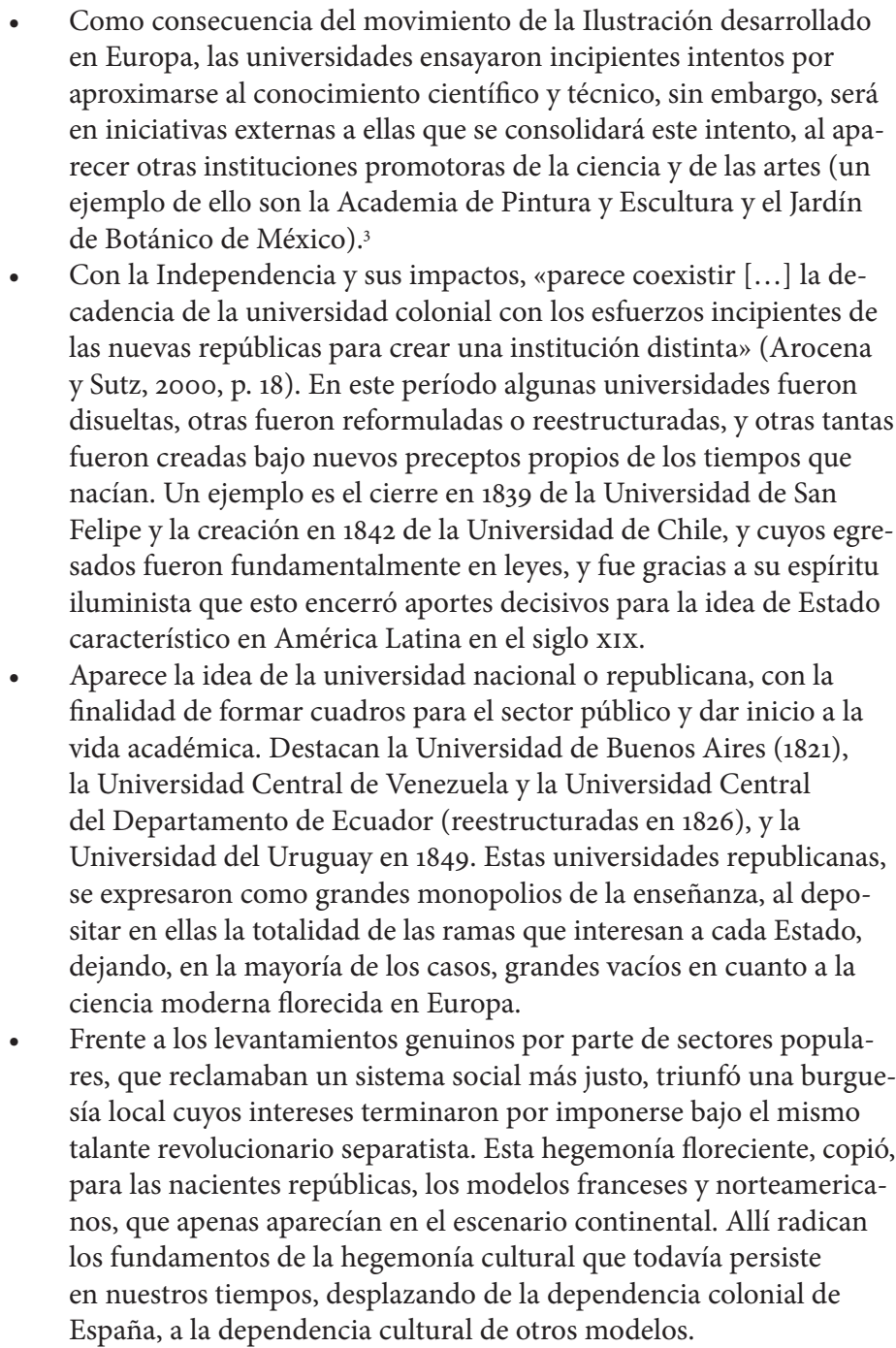 \\
\hline
\end{tabular}

3 Vale la pena en este punto llamar la atención sobre los trabajos de Tünnermann (1991) y de Steger (1974), en los cuales aparece una cronología y una tipología, respectivamente, ampliamente detalladas de las universidades hispanoamericanas. La primera de ellas fue la Universidad de Santo Domingo, en la Isla Española, en 1538, perteneciente al modelo de convento-universidad de Alcalá, y la última fue la de León de Nicaragua, en 1812, para un total de 32 fundaciones, muchas de las cuales no llegaron a funcionar realmente. Fueron las más importantes la de Lima y la de México, y sus modelos influyeron notoriamente en todo el continente, siendo precursoras de las universidades nacionales. 


\begin{tabular}{|c|c|}
\hline Período & Rasgos \\
\hline $\begin{array}{l}\text { Universidad } \\
\text { republicana } \\
\text { (siglos XVIII y } \\
\text { xIx) }\end{array}$ & $\begin{array}{l}\text { - El modelo de la universidad republicana será la universidad france- } \\
\text { sa-napoleónica de entonces, que propicia la educación politécnica } \\
\text { y profesional, separando la investigación de la formación. Se crean } \\
\text { academias e institutos dirigidos a la formación politécnica, y las } \\
\text { universidades pasan a la tutela del Estado principalmente orientadas } \\
\text { a la formación de los profesionales requeridos por el sector público, } \\
\text { lo que traduce, formar a la nueva elite burguesa y pequeño burguesa } \\
\text { requerida para fortalecer el nuevo orden social que se imponía en el } \\
\text { régimen republicano. }{ }^{4} \\
\text { Este modelo ofrece la formación politécnica de profesionales en } \\
\text { áreas técnicas, pero no necesariamente hacedores de ciencia ni } \\
\text { promotores de esta. Se promueve la formación de mano de obra } \\
\text { calificada de acuerdo a las demandas crecientes del capitalismo que } \\
\text { se fortalecía en el territorio. Las universidades quedan supeditadas, } \\
\text { bajo el mandato del Estado, a formar intelectuales mas no científi- } \\
\text { cos, pertenecientes fundamentalmente a las clases dominantes. }\end{array}$ \\
\hline $\begin{array}{l}\text { Universidad } \\
\text { contemporánea } \\
\text { (siglos xx y xxi) }\end{array}$ & $\begin{array}{l}\text { - Inicia el movimiento social y académico conocido como movi- } \\
\text { miento de Córdoba, surgido como respuesta al estado general en } \\
\text { que se encontraba la universidad en el continente, de la mano de la } \\
\text { situación política y social en general. } \\
\text { Este movimiento promueve como postulados fundamentales la } \\
\text { autonomía universitaria, }{ }^{5} \text { como necesidad de cobrar independencia } \\
\text { respecto del Estado, la gratuidad de la educación superior, el acceso } \\
\text { a la educación universitaria, la función social de la universidad y el } \\
\text { carácter popular necesario para las universidades. Todo ello circuló } \\
\text { con fuerza por todo el continente, dando lugar a diferentes reformas } \\
\text { en cada país, según sus propias condiciones sociohistóricas. }\end{array}$ \\
\hline
\end{tabular}

4 Creemos que este es un antecedente fundamental para el modelo de lo que conocemos como funciones universitarias (enseñanza, investigación y extensión), toda vez que el modelo napoleónico de universidad plantea un desarrollo escindido de las dos funciones iniciales, que eran la formación y la investigación, con especial énfasis en la formación de elites para fortalecer los sistemas de dominación, mediante la configuración de una clase pequeñoburguesa que se erige profesionalmente para legitimar y fortalecer a las burguesías locales y luego internacionales. Esta lógica, en muchos modos, sigue operando en las universidades del continente y las luchas al respecto van por el orden de revertirla ya que resulta constitutiva de nuestras instituciones universitarias.

5 En líneas anteriores dijimos que el antecedente de la noción de autonomía universitaria se ubicaba en la implantación del modelo de la Universidad de Alcalá de Henares, de corte jesuita, la cual promovía tal autonomía para imponer su lógica evangelizadora de formar misioneros, sin ninguna regulación por parte de reinados, virreinatos o gobiernos, ya que su fin último era la conquista espiritual mediante la acción autónoma de sus claustros, más de corte religioso que académico. Por su parte, en América Latina, comienza la lucha por la autonomía universitaria, con la intención de desprenderse de gobiernos autoritarios cuyos intereses estuviesen alejados de los intereses propios de la academia, intentando modificar la noción inicial de autonomía. 


\begin{tabular}{|c|c|}
\hline Período & Rasgos \\
\hline $\begin{array}{l}\text { Universidad } \\
\text { contemporánea } \\
\text { (siglos xx y xxI) }\end{array}$ & 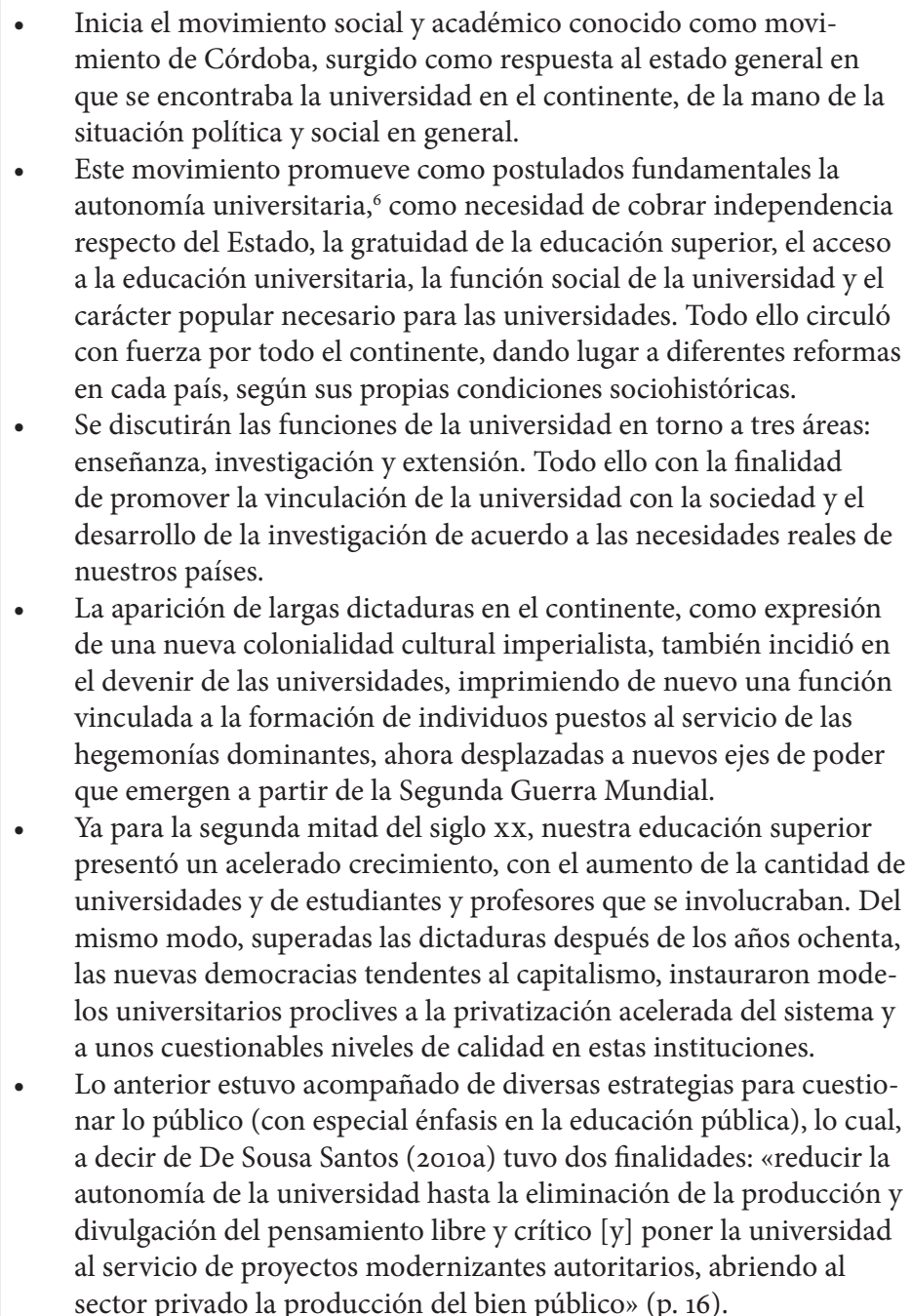 \\
\hline
\end{tabular}

6 En líneas anteriores dijimos que el antecedente de la noción de autonomía universitaria se ubicaba en la implantación del modelo de la Universidad de Alcalá de Henares, de corte jesuita, la cual promovía tal autonomía para imponer su lógica evangelizadora de formar misioneros, sin ninguna regulación por parte de reinados, virreinatos o gobiernos, ya que su fin último era la conquista espiritual mediante la acción autónoma de sus claustros, más de corte religioso que académico. Por su parte, en América Latina, comienza la lucha por la autonomía universitaria, con la intención de desprenderse de gobiernos autoritarios cuyos intereses estuviesen alejados de los intereses propios de la academia, intentando modificar la noción inicial de autonomía. 


\begin{tabular}{|c|c|}
\hline Período & Rasgos \\
\hline $\begin{array}{l}\text { Universidad } \\
\text { contemporánea } \\
\text { (siglos xx y xxi) }\end{array}$ & $\begin{array}{l}\text { - La imposición del modelo global neoliberalista generó una oferta de } \\
\text { servicios universitarios a modo de mercado que se vendían como } \\
\text { gran ventaja para el «desarrollo», en detrimento de la autonomía de } \\
\text { las universidades que entraban francamente en crisis por razones } \\
\text { diversas, entre ellas la financiera. Siguiendo con De Sousa Santos } \\
\text { (201oa), la autonomía pasó a ser "precaria y hasta falsa: porque } \\
\text { obligó a las universidades a buscar nuevas dependencias, de mucho } \\
\text { mayor costo que la dependencia del Estado y porque la concesión de } \\
\text { autonomía quedó sujeta a controles remotos estrictamente calibra- } \\
\text { dos por los ministerios de Hacienda y Educación» (p. 16). } \\
\text { Al mismo tiempo, una nueva corriente emergía en América Latina, } \\
\text { a partir de pensadores como Paulo Freire, Orlando Fals Borda, } \\
\text { Enrique Dussel, Oscar Jara. Bajo planteamientos como la educación } \\
\text { popular y la teología/filosofía de la liberación, se dan lugar diversas } \\
\text { manifestaciones críticas al modelo de colonialidad cultural que } \\
\text { aún se ha mantenido vigente, y promueven el desarrollo de otras } \\
\text { experiencias sociales y educativas. Estas iniciativas se mantuvieron } \\
\text { al margen de la educación formal, pero empezaron a expresarse en } \\
\text { propuestas alternativas a través de universidades de corte comunita- } \\
\text { rio en varios países que alcanzaron gobiernos no alineados al nuevo } \\
\text { orden mundial. } \\
\text { La creciente avanzada de las nuevas derechas en Latinoamérica en } \\
\text { los últimos años, está pugnando por deshacer, invisibilizar, anular } \\
\text { y desacreditar tales iniciativas, sobre todo por su gran potencial } \\
\text { para demostrar que existen otras vías, que es posible construir otros } \\
\text { modelos y porque dan cuenta de las enormes posibilidades que } \\
\text { tenemos para construir modelos propios de educación y de universi- } \\
\text { dad desde América Latina. }\end{array}$ \\
\hline
\end{tabular}

Fuente: elaboración propia.

Tenemos entonces, un escenario variopinto y complejo en el cual confluyen las luchas por defender la educación universitaria pública (y la educación pública en general), y por el acceso democrático y equitativo a las oportunidades de formación profesional. Existe un cuestionamiento al enfoque académico que sigue favoreciendo la formación en carreras propias del empleo asalariado, que privilegia una investigación basada en el statu quo y en conocimientos descontextualizados, y un modelo de extensión subalterno. Asimismo, se debaten intensamente los cambios que han de transitar los modelos educativos de nuestros países, en el entendido de que tales cambios van de la mano de los propios cambios socioeconómicos que vivimos y de

7 En este contexto, la lucha por la autonomía pasó a ser nuevamente un bastión. Antes lo habría sido para liberar a las universidades de las pretensiones de las dictaduras de la región; luego, se trata de marcar distancia con respecto a Estados puestos a los pies del neoliberalismo y de la crecida del capitalismo global, cuyos ímpetus privatizadores atentaban contra las universidades públicas con la firme intención de privatizar la educación universitaria, así como todos los servicios públicos en general. 
la postura que se asume ante el imperialismo y el capitalismo global, otorgando un claro talante político a los debates de esto que llamamos «lo universitario». ${ }^{8}$

Cabe destacar que el desarrollo de la extensión universitaria, también está vinculado con la historia misma de la universidad y de sus funciones. Si bien esta institución se crea con la función inicial de enseñar, ha ido adquiriendo formas diversas a medida que se han dado acontecimientos históricos que la han impactado directamente. Así, esta tercera función en las universidades de América Latina cobra sentido en la medida en la cual se reclama un saber que favorezca a los sectores más desventajados y, por ende, más llenos de necesidades, por lo que la extensión tendrá un indiscutible matiz político. Por ende,

concebimos a la extensión universitaria como el conjunto de actividades de colaboración entre actores universitarios y no universitarios, en formas tales que todos los actores involucrados aportan sus respectivos saberes y aprenden en un proceso interactivo orientado a la expansión de la cultura y a la utilización socialmente valiosa del conocimiento con prioridad a los sectores más postergados (Arocena, 2011, p. 11).

Si bien el planteamiento expuesto en la cita anterior es relativamente reciente y corresponde a lo que ha dado en llamarse la segunda reforma (en Uruguay), la extensión universitaria desde Córdoba comprende elementos que redundan en expresiones que se han distribuido por todo el continente con variados matices: compromiso social, deuda social, función social. Asimismo, en su problematización, se le ha denominado de diversas maneras para tratar con ello de imprimir matices diferenciadores (intención, proyecto comunitario, vinculación, entre otras). ${ }^{9}$

\section{Curricularización de la extensión en Uruguay y Venezuela}

Comenzamos aclarando que la selección de estos países para esta reflexión tiene que ver con el campo de experiencias de la autora, quien se ha desempeñado (con distintos tiempos de dedicación) en ambas instituciones en diversos roles, lo que ofrece insumos y vivencias para exponer estas consideraciones. Dicho esto, es importante definir lo que llamamos curricularización de la extensión y lo haremos a partir de palabras de Rodrigo Arocena (2011), quien expone:

La curricularización de la extensión puede ser entendida como asignarle unos pocos créditos en cada Plan de Estudios a algún curso de extensión, y chau. Semejante saludo a la bandera no implicaría avances sustantivos y más bien encapsularía a la extensión en el terreno reducido de unos pocos especialistas o vocacionales del asunto. No es eso lo que se propone, y no debería ser demasiado difícil comprenderlo. Se trata de

8 Al respecto puede verse lo expuesto por De Sousa Santos (2010a), y por Brunner y Ferrada Hurtado (2011).

9 Para conocer con más detalles la historia de la universidad uruguaya y su modelo de extensión, se recomiendan los libros de Arturo Ardao (2008) y Jorge Bralich (2007). Pero creemos que su evolución es consistente con lo expuesto hasta ahora concerniente a los modelos universitarios en América Latina a raíz de la colonización. 
explorar las diversas modalidades para la incorporación efectiva de la extensión al conjunto de la oferta educativa de la institución (p. 14).

A partir de lo dicho, entendemos la curricularización de la extensión como todo conjunto de iniciativas que promuevan las actividades cooperativas entre actores académicos y no académicos, generando intercambios de saberes y experiencias y otorgando respaldo crediticio, es decir, acreditación curricular en los planes de estudios de las diferentes carreras o programas de formación.

En el caso de Uruguay, estas iniciativas se orientan hacia el trabajo en prácticas y proyectos integrales, en el entendido de que allí se articulan las tres funciones básicas de la universidad (enseñanza, investigación y extensión), todas ellas orientadas a trabajar en territorio, interactuando con diferentes actores de la comunidad, movimientos sociales, abordando los problemas en búsqueda de soluciones, poniendo en diálogo y crítica constante entre otras, a las políticas públicas, permitiendo de este modo un espacio de construcción de ciudadanía.

Esto tiene expresión curricular en dos figuras concretas: los Espacios de Formación Integral (EFI) y una serie de unidades curriculares ofrecidas en los diversos planes de estudios de todas las carreras, vinculadas a la comprensión de la integralidad y al desarrollo de actividades en el medio, siendo los EFI, «dispositivos flexibles que se conforman a partir de múltiples experiencias educativas en diálogo con la sociedad, prácticas, cursos, talleres, pasantías, proyectos de extensión o investigación, asumiendo diferentes formas de reconocimiento curricular según las características de cada servicio universitario» (Tommasino y Rodríguez, 2011, p. 20).

Con el fin de colocar la universidad al servicio de la comunidad, los proyectos integrales devienen espacios transdisciplinarios de articulación de saberes y promoción de una transformación que incluye las tres funciones. Más allá de ello, la extensión se convierte en el eje central de dicha transformación al ser precisamente en ella donde se manifiestan las posibilidades creadoras de diversos colectivos de estudiantes, docentes y comunidades vinculadas.

Todos estos planteamientos forman parte de la Reforma que se desarrolla en la Universidad de la República desde 2007, consagrándose en una nueva Ordenanza de Estudios de Grado (Universidad de la República, 2011) que, entre otras cosas, coloca al estudiantado en el centro de los procesos, desplazándose de la mirada centrada en el docente, en la visión transferencista y bancaria de la educación y promoviendo una participación más activa y crítica de diversos sectores universitarios y sociales. Asimismo, se expresa en una revisión curricular que implicó la modificación de todos los planes de estudios, mismos que todavía hoy se encuentran en revisión y que cuentan con una flexibilidad amparada en la libertad de cátedra, por lo que se trata de un proceso aún en construcción, complejo y diverso, según la cantidad de docentes, áreas, unidades curriculares, disciplinas y posturas hagan vida en el mundo universitario. 
En otras latitudes, en 2004 se crea la Universidad Bolivariana de Venezuela, concebida como un "proyecto político emancipador, que debe estar orientado y contribuir con la construcción de una nueva hegemonía cultural, que tome distancia de las tendencias conservadoras de reproducción de modelos academicistas, y se acerque más bien, a un modelo histórico-crítico» (Hortegano, 2008, p. 8). Su proyecto quedó plasmado en un documento rector (Universidad Bolivariana de Venezuela, 2003) que, circunscrito a la Constitución recién reformulada en 1999, y en atención a los planes estratégicos de desarrollo del país, contribuyeran con la concreción de metas y objetivos vinculados no solo con garantizar el acceso a la educación universitaria a las poblaciones vulnerables y excluidas, sino con la formación de nuevos profesionales preparados para los procesos de transformación sociopolítica hacia modelos no capitalistas.

La UBV inició con una propuesta organizativa y curricular que cuestiona la lógica centrada en las tres funciones de la universidad, y pasa a proponer una estructura alternativa en la cual aparecen figuras como: integración socioeducativa, desarrollo socioacadémico, producción y recreación de saberes, vinculación sociocomunitaria. La propuesta se sustenta en un modelo curricular integrador, con planes de estudio que giran en torno a una Unidad Básica Integradora Proyecto (UBIP), la cual constituye un eje articulador de todo el mapa curricular,

... la cual está presente en todos los Trayectos y Tramos de todos y cada uno de los Programas de Formación de Grado. Esto con la finalidad de implementar la propuesta de la pedagogía de la praxis, en la cual, como ya se dijo anteriormente, se plantea la indivisible relación entre teoría y práctica como procesos complementarios, dialécticos [...] deviene espacio de encuentro entre [...] el conocimiento académico y las realidades comunitarias, las realidades de los colectivos, las necesidades de los sectores populares. Se convierte así en ese escenario que ha de garantizar el verdadero «diálogo de saberes» que propugna la UBV (Hortegano, 2018, p. 14).

En tanto unidad integradora, la UBIP articula el diseño curricular de las diferentes unidades curriculares según cada tramo (semestre) y trayecto (año), mismas que están pensadas para aportar elementos teóricos, epistemológicos y metodológicos para el desarrollo del trabajo en territorio que la UBIP implica. De esta forma, es fundamental el trabajo en colectivos docentes para planificar las acciones académicas e investigativas, las cuales deben tributar a la UBIP y al contexto en el cual se desarrolla.

Más adelante, como parte de un largo proceso de revisiones y reestructuraciones internas tanto organizativas como curriculares para fortalecer el trabajo colectivo y la UBIP, se reglamentarán las funciones del personal docente en torno a varios componentes y acciones que distan mucho de las tres funciones tradicionales; a saber:

1. Producción y recreación de conocimientos y saberes, que incluye: investigación, preparación de materiales de apoyo para encuentros didácticos; planificación y evaluación curricular; formación avanzada, educación continua. 
2. Gestión político-académica: dirección o coordinación educativa; planes, programas y proyectos académicos en contexto territorial.

3. Integración socioeducativa: interrelación sociocomunitaria y socioeducativa, corresponde a las distintas actividades que se desarrollan en los espacios comunitarios donde se desarrolla la UBIP; relación interactuante entre sujetos de la comunidad, organismos e instancias del Estado, estudiantes y docentes

4. Formación integral: tutorías, asesorías, seguimiento académico y Acompañamiento pedagógico, encuentros didácticos y desarrollo curricular en pro del proceso formativo de los estudiantes (UBV, 2016).

Como puede observarse, la curricularización de la extensión en este caso, tiene que ver con la constitución de un diseño curricular fundamentado en una unidad integradora orientada hacia el trabajo en territorio y en torno a la cual debe desarrollarse toda actividad de enseñanza y de investigación, es decir, que el trabajo territorializado es el eje del currículo y del desempeño académico e investigativo de estudiantes y docentes, convirtiendo a la extensión en el centro del currículo, pero una extensión reconfigurada, resemantizada y convertida en integración socioeducativa (ISE), la cual supone la transformación de todo el conjunto de prácticas de la universidad, convirtiéndola en el eje de la transformación universitaria. En atención al modelo de la UBV, la ISE pasó a definirse en los siguientes términos:

... una praxis resultante del proceso que permite hacer que la parte pase a reconocerse como constitución del todo social y geohistórico, que alude a una relación expresada en un proceso de trabajo en el que la universidad se vincula en una relación integral e integradora con la comunidad y el Estado, ${ }^{10}$ a partir de lo cual los saberes se encuentran en un solo espacio de formación-investigación y vinculación orgánica a las comunidades, cuyas necesidades pasan a ser objeto de trabajo compartido entre la universidad, el Estado y la comunidad misma, a través de sus actores principales: estudiantes, profesores, organizaciones sociales, consejos comunales, instituciones. Es a la vez el proceso mediante el cual la universidad, la comunidad y el Estado:

a) encuentran un objetivo común que los integra, a partir del reconocimiento de la unidad de lo diverso que representan las realidades locales enmarcadas en la realidad nacional; b) superan la separación en partes distintas, y c) en síntesis, avanzan juntos a construir una totalidad comprometida con la formación-investigación-acción-participativa-transformadora en el sentido del proyecto de nación (Córdova, 2012, p. 100).

10 Es importante no perder de vista que la UBV fue creada en medio de un contexto de transformaciones políticas y sociales en el país y como política pública para garantizar el acceso a la educación universitaria de los sectores excluidos, así como la formación de profesionales con perfiles idóneos para hacer posible el proceso de cambio que se iniciaba. Se inicia con un conjunto de programas de formación de grado (PFG) considerados estratégicos para el país en ese momento y se llevaron a toda la geografía nacional. Adicionalmente, el trabajo en territorio supone relaciones interinstitucionales y vínculo con las diversas políticas públicas en los diversos sectores con los cuales se vinculan los PFG. 
Dicho todo lo anterior, podemos puntualizar algunas similitudes concretas entre ambos modelos de extensión:

1. Ambos parten de la necesidad de atender demandas de sectores sociales concretos, históricamente desplazados y vulnerables, que usualmente no son vistos desde la academia.

2. Se coincide en el carácter transformador de la universidad más allá de la reproducción de modelos sociales que funcionan solo a favor de sectores privilegiados y de la ciencia moderna anclada en las academias.

3. Ambos modelos abordan el trabajo comunitario como elemento sustantivo, teniendo como pilar el diálogo de saberes y la ecología de saberes (De Sousa Santos, 2010b).

4. Se promueve en los dos casos la sistematización de experiencias como alternativa metodológica para el rescate de saberes populares.

5. La incorporación de las metodologías participativas en los dos modelos, supone el diagnóstico participativo como parte del abordaje metodológico.

6. Se problematiza la evaluación en el marco de metodologías emergentes para el trabajo en territorio, que obliga a pensar la evaluación educativa desde otros lugares.

7. La extensión es la función sustantiva de la universidad en ambos casos, aunque su forma de obtener reconocimiento curricular es muy distinta.

8. En ambos modelos se parte de una problematización de las funciones universitarias.

9. Existe en las dos visiones, una vinculación con las políticas públicas, ya que se busca «ayudar a consolidar y profundizar la organización de los sujetos que participan o son beneficiarios de las políticas públicas» (Tommasino y Rodríguez, 2011, p. 26), en el entendido de que es en la relación con tales políticas que puede promoverse la construcción de ciudadanía y problematizar las formas sociales de reproducción de la dominación en la medida en que se trata de la organización de colectivos.

Por otra parte, la diferencia fundamental entre los dos modelos, consiste en la forma cómo se ha curricularizado la extensión, ya que, en un caso, se trata de diversas unidades curriculares, programas, proyectos y espacios integrales, mientras que en el otro consiste en una unidad curricular integradora que recorre todo el plan de estudios y en torno a la cual se articulan todas las demás actividades académicas y de investigación. Pese a esta diferencia, se trata igualmente de promover el trabajo en territorio como elemento sustantivo del currículo con la finalidad de producir saberes con y para las comunidades y colectivos. 


\section{Consideraciones finales: para decolonizar la extensión}

La decolonialidad como categoría teórica, es una propuesta elaborada por un grupo multidisciplinario de pensadores/as latinoamericanos/as11 que conforman lo que ha dado en llamarse Grupo Modernidad/Colonialidad.12 Dicho grupo elabora una serie de propuestas teóricas, metodológicas y epistemológicas que apuntan a superar la dominación que sigue operando sobre los pueblos oprimidos del mundo. En palabras de Castro-Gómez y Grosfoguel (2007):

El concepto «decolonialidad» [...] resulta útil para trascender la suposición de ciertos discursos académicos y políticos, según la cual, con el fin de las administraciones coloniales y la formación de los Estados-nación en la periferia, vivimos ahora en un mundo descolonizado y poscolonial. Nosotros partimos, en cambio, del supuesto de que [como consecuencia de] la división internacional del trabajo entre centros y periferias, así como la jerarquización étnico-racial de las poblaciones, formada durante varios siglos de expansión colonial europea [...] Asistimos a una transición del colonialismo moderno a la colonialidad global... (p. 13).

Esta colonialidad global se expresa en una colonialidad del ser, ${ }^{13}$ del saber ${ }^{14} y$ del poder $^{15}$ que permite perpetuar las formas hegemónicas que imperan en el mundo globalizado. De allí que, la decolonización epistemológica, deviene posibilidad de superar las formas de colonialidad mencionadas y trascender la dominación histórica que hemos vivido los pueblos colonizados, ${ }^{16}$ en el entendido de que la colonialidad, en todas sus expresiones, es

... uno de los elementos constitutivos y específicos del patrón mundial de poder capitalista. Se funda en la imposición de una clasificación racial-étnica de la población del mundo como piedra angular de dicho patrón de poder, y opera en cada uno de los planos, ámbitos y dimensiones, materiales y subjetivas, de la existencia cotidiana y a escala social (Quijano, 2020).

Desde esta postura, se cuestiona una modernidad que pasa a situarse como centro del sistema-mundo y que tiene pretensiones de universalización de la cultura europea.

11 Este grupo, que ha venido creciendo en las dos últimas décadas, cuenta con nombres como: Walter Mignolo, Catherine Walsh, Aníbal Quijano, Santiago Castro-Gómez, Edgardo Lander, Javier Sanjinés, Nelson Maldonado Torres, María Lugones, Ramón Grosfoguel, Agustín Lao-Montes, Fernando Coronil, Enrique Dussel, Zulma Palermo, Arturo Escobar, Fernando Coronil, Boaventura de Sousa Santos (quien desarrolla las Epistemologías del Sur), entre otros y otras.

12 Una genealogía útil para conocer los perfiles, intereses y producciones de este grupo, aparece en Castro-Gómez y Grosfoguel (2007).

13 La colonialidad del ser nos aproxima a la neocolonialidad más entendida como fenómeno cultural y que incluye las discusiones en torno al sujeto moderno, la otredad y la identidad. La consideramos a partir de lo desarrollado por Mignolo $(1995,2015)$ y Maldonado Torres (2007).

14 Incorporamos la colonialidad del saber a partir de Restrepo y Rojas (2010), vinculado con la producción de un conocimiento limitado a la reproducción de la episteme colonial.

15 El concepto de colonialidad del poder es desarrollado por Quijano (1992, 2000) a partir de la idea de raza como eje, de allí una organización del poder global a partir de la división racial del trabajo.

16 Ver en Dussel (2015) una descripción de las distintas corrientes teóricas que forman parte de estas propuestas y sus respectivas explicaciones. 
Este modelo, según Dussel (2015), cuenta con «cuatro aspectos de un mismo proceso y determinaciones simultáneas» (p. 332), a saber: modernidad, eurocentrismo, colonialismo y capitalismo. A estos cuatro añadiremos el patriarcado como elemento constitutivo de su encuadre epistémico.

Pensar la integralidad decolonialmente, después de todo lo dicho, supone partir de varias problematizaciones fundamentales: 1) ¿Desde dónde se inicia la producción de conocimiento? ¿Cuál es el lugar que este conocimiento ocupa? ¿Qué conocimiento queremos construir y para qué? ¿Qué lugar ocupará este conocimiento en la sociedad en la cual se produce? Esto entraña una discusión en torno a la escisión entre el saber científico/académico y los saberes colectivos y populares, y a la colonialidad del saber; 2) ¿Qué lugar ocupan las personas en esta producción de conocimiento? ¿Cuál es el sujeto que piensa y que se debe pensar? ¿Cómo se configuran las identidades y la intersubjetividad en este conocimiento? Y aquí se remite a la colonialidad del ser, $y$, 3) ¿Cuáles relaciones operan y deben operar entre las personas que participan en esta producción de conocimientos? ¿Se trata aún de una relación sujeto-objeto? ¿Cómo se establecerán los vínculos metodológicos y epistemológicos? Y ello deriva a la colonialidad del poder. La colonialidad del ser, del saber y del poder opera para generar una lógica relacional asimétrica.

Recordemos que todos los esfuerzos de la educación universitaria en América Latina (esbozados en la primera parte de este artículo) tienen vinculación con el profundo sentido emancipador que hace vida en nuestros pueblos y con la necesidad de superar una larga historia de dominaciones, muertes y opresiones. Por ello, luce adecuado pensar en la decolonialidad como opción para dar fundamentos a unas prácticas y teorías de lo nuestro y con especial énfasis en la necesidad de edificar nuevos modelos sociales no eurocéntricos ni universalistas.

Asimismo, para decolonizar la extensión, sus modelos y prácticas, es preciso rehacer los cimientos mismos de la esencia universitaria, que ha estado basada en abstraerse de la realidad para elaborar un saber "puro», universal y objetivo, que son los pilares de la ciencia moderna/colonial. Permeada con esta lógica, la universidad se erigió como una entidad aislada de la realidad de los sectores dominados, considerando el saber producido en contexto como un saber subalterno, menor.

De igual modo, situarse en una mirada decolonial de la extensión es poner el interés en dar a pensar las potencialidades que tiene América Latina y todo pueblo oprimido, para producir modelos propios de educación y de universidad; así como destacar que es imperativo decolonizar el saber y el ser para poder reconfigurar las prácticas de extensión más allá de la lógica extractivista del conocimiento académico, que reduce a categorías teóricas y certificaciones los saberes populares, aniquilando su potencial transformador. Decolonizar la extensión es también combatir la idea de universidad clasista, formadora de una pequeña burguesía desclasada. 
En tal sentido es conveniente tener en cuenta que nuestro continente no ha dejado de sufrir los ataques colonizadores del norte global. Superadas las colonias territoriales, queda la colonialidad cultural y la dominación económica que, bajo la globalización neoliberal sigue imponiéndose, ahora en la forma de nuevas derechas y con un empuje importante por parte del capital privado de nuevo sobre los bienes públicos

La decolonización de la extensión implica la decolonización misma de la universidad y su rol ante las realidades latinoamericanas, ya que, si bien es cierto que se avanza en modelos innovadores como los descritos en estas páginas, aún queda mucho por hacer. Las formas a través de las que se ha curricularizado la extensión trae consigo varias dificultades que mantienen vivo el debate sobre estas prácticas y que hacen pensar que es necesario profundizar en ello:

1. En el modelo de la Udelar, la duración de un EFI, unidad curricular o proyecto, suele tener plazos acotados cuando en realidad las dinámicas concretas de comunidades, instituciones, colectivos, no se restringe a los tiempos universitarios organizados en semestres lectivos. Esto hace que, muchas veces, lo que se hace se limite a un recorte de la realidad o se terminen desvirtuando las actividades desarrolladas.

2. En el modelo de la UBV, la masividad, la incorporación de profesionales con perfiles no adecuados para desarrollar la UBIP, la poca experticia en metodologías participativas, ha jugado al desgaste de comunidades, colectivos de estudiantes, docentes e instituciones, que se limitan a cumplir protocolos académicos y crediticios sin mayor impacto en el territorio.

3. En muchos casos, los colectivos o comunidades esperan que quienes llegan de la universidad les ayuden a resolver determinados problemas $y$, toda vez que esto no es posible porque excede los roles de los cursos, queda la comunidad decepcionada y desgastada.

4. La extensión no ha superado la lógica de la división social impresa en las universidades, lo cual es propio de la lógica moderna-colonial-capitalista. De esta forma, aún se aprecian prácticas de extensión donde la figura de los/as actores/as universitarios/as sigue siendo la de expertos/as que van a la «comunidad» a llevar un conocimiento válido y verdadero.

5. Esto implica que el ejercicio de producción de conocimiento sigue siendo el de una ciencia extractivista y utilitaria que se vale de las informaciones que se obtienen en el territorio para cumplir con los requerimientos y protocolos de una academia que solo cumple formalismos.

6. La lógica detrás de la palabra extensión supone una universidad que va hacia afuera donde están los otros a llevar conocimiento. Ello supone la legitimación de una línea abisal, en palabras de De Sousa Santos (2010b), 
línea esta que sigue dejando en algún lugar más allá del yo, a los sujetos del conocimiento.

7. La inminente relación entre extensión e investigación, pasa por una lógica epistemicida ${ }^{17}$ propia de la colonialidad del saber, donde los resultados de las actividades desarrolladas quedan la mayoría de las veces solo en favor de la academia, más vista como entelequia que como una institución al servicio de una sociedad precaria.

8. Es valioso reconocer que la dinámica conservadora de enseñar e investigar, no ha abandonado las universidades latinoamericanas, a pesar de ser pioneras en la preocupación por el compromiso social que debe atenderse desde la academia. Pensar las universidades al servicio de la sociedad no ha sido suficiente, mientras siga existiendo un currículo oculto que, solapado detrás de discursos políticamente correctos, siguen apuntalando a la acumulación de certificados en las hojas de vida de cada docente, y a la lógica del status académico más que a la del trabajo en territorio.

Ante estas dificultades, no concebimos la actividad universitaria de otra forma que no sea mediante la integralidad de funciones, pero entendemos tal integralidad en términos bastante amplios, en los cuales es preciso repensar nuevamente los currículos de cada universidad en atención a contemplar otros fundamentos axiológicos y teleológicos. Todo ello solo es posible si existe un debate profundo acerca del modelo de sociedad que se aspira y, por ende, al perfil de cada profesional que se espera egresar. Del mismo modo, cada docente, cada unidad curricular, debe trascender la lógica transmisionista de contenidos, la postura de poder implícita en la figura del docente; y avanzar hacia otras formas de relacionarse con sus estudiantes, con la sociedad y con los propósitos que se plantea la universidad pública hoy.

La decolonización de la extensión no solo pasa por la ecología y el diálogo de saberes, sino que implica que, como universitarios/as, logremos reconocernos como agentes de cambio y no como dueños/as de ningún saber. Logremos pensarnos como parte de la otredad que está del otro lado de la línea abisal, en tanto colonizados/as y subordinados/as por poderes globales del nuevo orden mundial, colonial, patriarcal y capitalista. Así, miraremos la enseñanza universitaria como un proceso recursivo y constructivo donde la investigación y la extensión dejan de ser funciones separadas, y

17 Para pensar el epistemicidio y la violencia epistémica, se recomienda leer a Césaire (1978), quien explica cómo la historia de los pueblos dominados pasa de la colonización a la colonialidad, es decir, de la ocupación territorial mediante muerte, violencia y saqueos, a la colonialidad cultural mediante estrategias burguesas centradas en la «idea» de superioridad y de progreso civilizatorio que termina por anular toda memoria, todo rastro y toda episteme de lo que ha sido en realidad cada pueblo colonizado, operando un desplazamiento epistémico gracias al cual el dominado reclama la implementación de las estrategias del colonizador por creerlas mejores, superiores y oportunas. Desde esta idea, el académico deviene colonizador para el cual siempre prevalecen sus propias ideas por creerlas superiores y legítimas. Este planteamiento aparecerá en innumerables escritos de De Sousa Santos, quien lo trae a la actualidad. 
pasarán todas a ser parte natural del quehacer universitario por parte de todos/as sus actores/as, ya que todos/as somos comunidad.

El núcleo de la decolonización de la extensión no es demostrar que cada actividad de extensión incluye de forma integral a la enseñanza y la investigación. A estas alturas es difícil negar que en tales actividades se construye un saber valioso socialmente y se puede investigar con validez y aportar grandes conocimientos a la academia y a la sociedad. La verdadera meta radica en transformar la enseñanza y la investigación hacia un enfoque integrador, y no solo hacia el desarrollo de actividades encerradas disciplinariamente, o secuestradas por los métodos clásicos de la academia oficial, que siguen enclaustrados y de espaldas a la sociedad, como si el puro saber teórico o el puro dato estadístico en efecto pudieran mejorar las condiciones de miles de hombres, mujeres, niños y niñas que han padecido históricamente todas las formas de negación, exclusión y segregación que el mundo occidental ha producido en su lógica clasista.

Desde esta perspectiva, como señala Eduardo Álvarez Pedrosian (2011), «es necesario poner en juego siempre las condiciones de clase, género, étnicas, sociales y culturales que están implícitas en quienes se embarcan en un proceso de creación de conocimiento» (p. 66), por ende, el reto es convertir la extensión en el centro de las actividades curriculares, comenzando por una universidad transversalizada por la impronta social, pero donde las prácticas diarias cuenten con una sólida fundamentación ontológica, axiológica, epistemológica, filosófica y pedagógica congruentes con los nuevos fines. Donde la autonomía y la libertad de cátedra no se conviertan en etiqueta de ocasión para justificar el anacronismo, las prácticas ortodoxas y las lógicas coloniales que han imperado en la enseñanza universitaria por siglos.

Se trata de una «naturalización de la extensión», como dice Arocena (2011). Para ello debe superarse la visión subalterna que tiene la academia de todo aquello que tiene matiz comunitario o de lo que se construye fuera del claustro, ya que ello ha obligado a la extensión a obtener elementos de validación y credibilidad con la necesidad de «convencer» a los académicos de que lo que se hace en territorio también es investigación y también es conocimiento científico.

Muchos años de debate acerca de métodos y saberes emergentes, no han sido suficientes para demoler la lógica ortodoxa que permea nuestras universidades. Aun cuando proclamamos grandes discursos por la emancipación, las prácticas mínimas, cotidianas, internas de cada universidad, siguen reproduciendo la misma lógica centrada en ciertos privilegios para algunos, limosnas para otros.

No es gratuito que se piense en la extensión como el eje impulsador de la transformación universitaria, ya que es desde dicho eje que se puede promover una enseñanza distinta, no solo centrada en contenidos, sino pensada en función de otros logros que trasciendan la acumulación teórica y la repetición de citas y autores. Tal conocimiento tendría que cobrar sentido en las posibilidades de aplicación a situaciones sociales 
concretas y en la medida en que favorezcan procesos colectivos de transformación. Seguimos creyendo que tal transformación es posible, pero solo en el marco de las universidades públicas de América Latina, siempre y cuando estas dejen su lugar de privilegios para privilegiados y agachen la cabeza para dejar hablar a los excluidos.

\section{Referencias}

Álvarez Pedrosian, E. (2011). Crear, aprender y compartir: apuntes epistemológicos sobre la integralidad. Cuadernos de Extensión, 1: Integralidad: tensiones y perspectivas (pp. 61-83). Montevideo: CSEAm, Universidad de la República. Recuperado de https://www.extension.udelar.edu.uy/ wp-content/uploads/2017/11/Cuaderno-n\%C2\%Bo1-integralidad.pdf

Ardao, A. (2008). La Universidad de Montevideo: su evolución histórica. Montevideo: Universidad de la República. Recuperado de https://anaforas.fic.edu.uy/jspui/bitstream/123456789/57348/1/ardao_-_ universidad_montevideo.pdf

Arocena, R. (2011). Curricularización de la extensión: ¿por qué, cuál, cómo? Cuadernos de Extensión, 1: Integralidad: tensiones y perspectivas (pp. 10-18). Montevideo: CSEAM, Universidad de la República. Recuperado de https://www.extension.udelar.edu.uy/wp-content/uploads/2017/11/ Cuaderno-n\%C2\%Bo1-integralidad.pdf

Arocena, R., y Sutz, J. (2000). Universidad latinoamericana del futuro. Ciudad de México: Unión de Universidades de América Latina. Recuperado de https://campus.fundec.org.ar/admin/archivos/ EAD\%20-\%20Arocena\%20y\%20Sutz\%2O-\%20An\%C3\%A1lisis\%2oprospectivo.pdf.

BRALICH, J. (2007). La extensión universitaria en el Uruguay: Antecedentes y desarrollo en la Universidad de la República desde sus inicios hasta 1996. Montevideo: CSEAM, Universidad de la República. Recuperado de https://www.extension.udelar.edu.uy/wp-content/uploads/2017/11/La-extensionuniversitaria-en-el-uruguay-Bralich-1.pdf.

BRUNNER, J. J. (1990). Educación superior, investigación científica y transformaciones culturales en América Latina. Santiago de Chile: BID-SECAB-CINDA.

Brunner, J. J., y Ferrada Hurtado, R. (Eds.). (2011). Educación superior en Iberoamérica: Informe 2011. Santiago de Chile: Centro Interuniversitario de Desarrollo-Universia.

CAstro-Gómez, S., y Grosfoguel, R. (2007). El giro decolonial: reflexiones para una diversidad epistémica más allá del capitalismo global. Bogotá: Siglo del Hombre Editores-Universidad CentralIESCO-Pontificia Universidad Javeriana-Instituto Pensar. Recuperado de http://www.unsa.edu.ar/ histocat/hamoderna/grosfoguelcastrogomez.pdf.

CÉSAIRE, A. (1978). Discurso sobre o colonialismo (Trad. Noèmia de Sousa). Lisboa: Livraria Sá Da Costa Editora

Córdova, M. Y. (2012). Integración socioeducativa como eje estratégico para la transformación universitaria. Caracas: OPSU/CNU.

De Sousa SAntos, B. (2010a). La universidad en el siglo xxi: para una reforma democrática y emancipatoria de la universidad. Montevideo: CseAm, Universidad de la República-Ediciones Trilce. Recuperado de https://www.extension.udelar.edu.uy/wp-content/uploads/2017/11/La-universidaden-el-siglo-Xxi.pdf.

De Sousa Santos, B. (2010b). Descolonizar el saber, reinventar el poder. Montevideo: CSEAM, Universidad de la República- Ediciones Trilce. Recuperado de http://www.boaventuradesousasantos.pt/media/ Descolonizar\%2oel\%2osaber_final\%20-\%20C\%C3\%B3pia.pdf.

Dussel, E. (2015). Filosofías del sur: descolonización y transmodernidad. Ciudad de México: Akal. 
Hortegano, R. (2008). Enfoque de género y educación emancipadora: reflexiones en torno a su vinculación con Proyecto y la UC bases del conocimiento en el PFG Gestión Social del Desarrollo Local. (Trabajo presentado para optar al escalafón de profesor asistente en la Universidad Bolivariana de Venezuela).

JimÉnEZ, E. (2007). La historia de la universidad en América Latina. Revista de la Educación Superior, 36(141), 169-178. Recuperado de https://www.researchgate.net/ publication/28307738_La_historia_de_la_universidad_en_America_Latina

Maldonado Torres, N. (2007). Sobre la colonialidad del ser: contribuciones al desarrollo de un concepto. En S. Castro-Gómez y R. Grosfoguel (Comps.), El giro decolonial: reflexiones para una diversidad epistémica más allá del capitalismo global. Bogotá: Siglo del Hombre EditoresUniversidad Central-IESC-Pontificia Universidad Javeriana-Instituto Pensar.

Mignolo, W. (1995). Decires fuera de lugar: sujetos dicentes, roles sociales y formas de inscripción. Revista de crítica literaria latinoamericana, 11, pp. 9-32.

Mignolo, W. (2008) La opción de-colonial: desprendimiento y apertura. Un manifiesto y un caso. Tabula Rasa, 8, 243-281. Recuperado de http://www.revistatabularasa.org/numero-8/mignolo1.pdf

Mignolo, W. (2015). Trayectorias de re-existencia: ensayos en torno a la colonialidad/decolonialidad del saber, el sentir y el creer. Bogotá: Universidad Distrital Francisco José de Caldas.

Quijano, A. (1992). Colonialidad y modernidad/racionalidad. Perú Indígena, 13(29), 11-20. Recuperado de https://www.lavaca.org/wp-content/uploads/2016/o4/quijano.pdf

Quijano, A. (200o). Colonialidad del poder, eurocentrismo y América Latina. En E. LANder (Comp.) La colonialidad del saber: eurocentrismo y ciencias sociales. Perspectivas latinoamericanas. Buenos Aires: Clacso. Recuperado de http://bibliotecavirtual.clacso.org.ar/ar/libros/lander/quijano.rtf

Quijano, A. (2020). Colonialidad del poder y clasificación social. En D. Assis (Comp.). Quijano, Aníbal. Cuestiones y horizontes: de la dependencia histórico-estructural a la colonialidad/descolonialidad del poder. Buenos Aires: Clacso; Lima: Universidad Nacional Mayor de San Marcos. Recuperado de http://biblioteca.clacso.edu.ar/clacso/se/20140424014720/Cuestionesyhorizontes.pdf

Restrepo, E., y Rojas, A. (2010). Inflexión decolonial: fuentes, conceptos y cuestionamientos. Popayán: Universidad del Cauca-Instituto de Estudios Sociales y Culturales Pensar. Recuperado de http:// www.ram-wan.net/restrepo/documentos/Inflexion.pdf

Steger, H.-A. (1974). Perspectivas para la planeación de la enseñanza superior en América Latina. Anuario de Estudios Latinoamericanos, (4).

Tommasino, H., y Cano, A. (2016). Modelos de extensión universitaria en las universidades latinoamericanas en el siglo xxI: tendencias y controversias. Universidades, (67), pp. 7-24. Recuperado de https://pim.udelar.edu.uy/wp-content/uploads/sites/14/2017/o4/Modelos-de-extensi\%C3\%B3nuniversitaria-en-las-universidades-latinoamericanas-en-el-siglo-XxI-tendencias-y-controversias.Tommasino-y-Cano.pdf.pdf

Tommasino, H., y Rodríguez, N. (2011). Tres tesis básicas sobre extensión y prácticas integrales en la Universidad de la República. Cuadernos de Extensión, 1: Integralidad: tensiones y perspectivas (pp. 19-42). Montevideo: CSEAM, Universidad de la República. Recuperado de https://www.extension. udelar.edu.uy/wp-content/uploads/2017/11/Cuaderno-n\%C2\%Bo1-integralidad.pdf

Tünnermann, C. (1991). Historia de la universidad en América Latina: de la época colonial a la Reforma de Córdoba. San José de Costa Rica: EDUCA. Recuperado de https://www.enriquebolanos.org/ media/publicacion/2980.pdf

Universidad Bolivariana de Venezuela (2003). Documento rector. Caracas: ubv. Recuperado de https://antonifrank.files.wordpress.com/2009/05/documento-rector-ubv1.pdf 
Universidad Bolivariana de Venezuela, Consejo Universitario (2016, noviembre 16). Normativa para oferta y carga académica. (Resolución n. $\left.{ }^{\circ} \mathrm{CU}-14-06-2016\right)$.

Universidad de la República, Consejo Directivo Central (2011, agosto). Ordenanza de estudios de grado y otros programas de formación terciaria (Resolución $n .^{\circ} 3$ del 2 de agosto y resolución n. ${ }^{\circ}$ 4 del 30 de agosto). Recuperado de https://www.cse.udelar.edu.uy/wp-content/uploads/2013/12/ documento_ordenanza_de_grado_corregida_paginas_simples.pdf. 